\title{
PREPARATION AND CHARACTERIZATION OF GLIADIN NANOPARTICLES FOR BRAIN DRUG DELIVERY
}

\author{
ANKIT DUBEY NAVENEET DUBEY*, SUMAN RAMTEKE, DEEPTI JAIN \\ School of Pharmaceutical Sciences, Rajiv Gandhi Proudyogikivishwavidyalaya, Bhopal - 462 033, Madhya Pradesh, India. \\ Email: naveneet@gmail.com
}

Received: 26 February 2018, Revised and Accepted: 14 April 2018

\begin{abstract}
Objectives: The aim of the present work is to prepare and evaluate nasal controlled release gliadin nanoparticles of sumatriptan for antimigraine activity to improve patients' compliance by simplifying its administration, improving its therapeutic effect, and reducing its dose-related side effects
\end{abstract} through brain targeting.

Methods: Sumatriptan containing gliadin nanoparticles were prepared by desolvation method using Pluronic F-68 as a stabilizing agent.

Results: The results show that this method is reproducible very easy and led to the efficient entrapment of drug as well as the formation of spherical particles ranging from 1 to $181 \mathrm{~nm}$, respectively. The controlled release behavior of gliadin nanoparticles was evaluated both in phosphate buffer saline $7.4 \mathrm{pH}$ and in phosphate buffer $6.8 \mathrm{pH}$, respectively, at $37 \pm 1^{\circ} \mathrm{C}$. Their brain targeting properties were determined by the nasal route through in vitro and in vivo methods.

Conclusion: The optimized nanoparticles showed higher release rate than other marketed formulation like a tablet, nasal spray this may be due to nano size of the particles, glutaraldehyde was added to this system that increased hardness and F-68 increased the stability of nanoparticles .tablet, nasal spray this may be due to nano size of the particles, glutaraldehyde was added to this system that increased hardness and F-68 increased the stability of nanoparticles. The in vivo results showed that within 15 min maximum concentration of sumatriptan was observed in brain as compare to plasma which could be due to small particle size and lipophilic nature of gliadin nanoparticles.

Keywords: Blood-brain barrier, Nasal drug delivery, Central nervous system, Nanotechnology, Nanoparticles, Brain targeting.

(C) 2018 The Authors. Published by Innovare Academic Sciences Pvt Ltd. This is an open access article under the CC BY license (http://creativecommons. org/licenses/by/4. 0/) DOI: http://dx.doi.org/10.22159/ajpcr.2018.v11i7.25478

\section{INTRODUCTION}

Migraine is characterized by episodes of head pain that is often throbbing and frequently unilateral and may be severe. In migraine without aura, attacks are usually associated with nausea, vomiting, or sensitivity to light,sound, or movement.When untreated, these attacks typically last 4 to 72 hours [1]. Migraine is a chronic neurological disorder characterized by recurrent moderate-to-severe headaches often in association with a number of autonomic nervous system symptoms [2]. Migraine is a typical endless multifactorial neuromuscular issue ordinarily portrayed by intermittent crippling assaults of an extreme cerebral pain autonomic sensory system brokenness and in up to $33 \%$ of patients neurological atmosphere side effects. Ergot subordinates used to be the main particular treatment for migraine assault in spite of the fact that they had numerous constraints enhanced comprehension of the neurobiology of migraine, and 5 HT receptors have brought about another class of specific 5HT agonist known as the triptans [3]. Beginning with sumatriptan in 1993, the intro-duction of the triptans provided an important oppor-tunity for improved headache management. 13 Educa-tional efforts directed at health care professionalsand individuals with headache were started to raiseawareness of the clinical manifestations and burdenof migraine as well as the availability of effectivetreatments. In the context of these developments, thepatterns of health care use for migraines may havechanged since 1989 [4]. These mixes have three putative principle systems of antimigraine activity which are presumably essentially intervened by a $5 \mathrm{HT} 1 \mathrm{~B} / 1 \mathrm{~T}$ receptor agonist action cranial vasoconstriction fringe neuronal hindrance and restraint of transmission through second-arrange neurons of the trigeminocervical complex. The relative importance of each of these mechanisms remains uncertain.In comparison with ergots, triptans have several distinct advantages. These include selective pharmacology, simple and consistent pharmacokinetics, evidence-based prescribing instructions, high efficacy, modest side-effects, and a wellestablished safety record (8). They are, however, like ergot derivatives, also contraindicated in the presence of cardiovascular disease. Despite the higher price, triptans are preferred over ergots in the majority of patients [3]. Late clinical investigations have demonstrated that sumatriptan is highly effective in the treatment of a migraine how much sumatriptan acts in such of obsessive situation; since the agony of a migraine is probably going to be a vascular inception, it appears to be sensible to recommend that its essential site of activity is at the level of the vein divider. A non-particular central pain-relieving activity is impossible found in animals, examines greatly high measurements of sumatriptan, and needs pain relieving movement; it is, however, likely that sumatriptan likely lifts the agony of migraine by building extended and edematous cranial veins. Sumatriptan is a vasoconstrictor of certain cranial vein in vitro prominently dosage of intracranial birthplace and the 5 HT1-like receptor that intercedes vasoconstriction which is confined transcendently on cerebral vessel from the two animals and people; however, animals and human examinations in vivo show that sumatriptan does not alter cerebral blood flow [5]. Sumatriptan being a prototype triptan drug is commonly used for therapeutic management of migraine. It mainly acts as an agonist at 5-hydroxytryptamine (5-HT1B/1D) receptor of serotonin (5-HT). 5-HT1B/1D receptor is a pre-synaptic receptor at serotonergic neurons and when bound by agonist inhibits the release of serotonin from serotonergic neurons. Hence, sumatriptan inhibits the release of serotonin from the serotonergic neurons [6].

\section{MATERIALS AND METHODS}

Sumatriptan was obtained as a gift sample from Mylan Laboratory, Hyderabad, and gliadin was obtained from MP Biomedicals. All other reagents were of analytical grade. 
Preparation of sumatriptan-loaded gliadin nanoparticulate system

The gliadin nanoparticles were prepared by a desolvation procedure as described by Ramteke et al. [7]. Briefly gliadin and sumatriptan were dissolved in $20 \mathrm{ml}$ of ethanol:water phase $(7: 3 \mathrm{v} / \mathrm{v})$, and this solution was poured into a physiological saline phase $(\mathrm{NaCl} 0.9 \% \mathrm{w} / \mathrm{v}$ in water) containing $0.5 \%$ pluronic F-68 as a stabilizer using programmable digital homogenizer (Omni pdh programmable digital homogenizer). Nanoparticles were hardened by the addition of $2 \mathrm{ml}$ of glutaraldehyde and stirred for $2 \mathrm{~h}$ at room temperature. Then, ethanol was eliminated by evaporation under the reduced pressure, and resulting nanoparticles were purified by centrifugation at $15,000 \mathrm{rpm}$ for $1 \mathrm{~h}$ in an ultracentrifuge (Beckman Coulter Optima Centrifuge). The supernatant was removed, and the pellets were resuspended in water. The suspension was passed through a $0.45 \mu \mathrm{m}$ pore size membrane filter. Finally, the nanoparticles were freeze-dried using $5 \%$ glucose solution as a cryoprotector.

\section{RESULTS AND DISCUSSION}

Percentage drug entrapment and percentage recovery

The percentage drug entrapment and percentage recovery were determined using the following equations:

\%Drug entrapment=Mass of drug in nanoparticles/Mass of drug used in the formulation $\times 100$

$\%$ Nanoparticles recovery (\%yield)=Concentration of drug in nanoparticles/Concentration of nanoparticles recovered $\times 100$

An appropriate amount of freeze-dried gliadin nanoparticles was digested with a minimum amount of ethanolic solution (water: ethanol in 7:3 ratios). The digested homogenates were centrifuged at 15,000 rpm for $30 \mathrm{~min}$, and the supernatant was analyzed for drug entrapment. The sumatriptan entrapment was measured at $227 \mathrm{~nm}$ using Shimadzu 1700 ultraviolet (UV)/visible spectrophotometer; the percentage drug entrapment and percentage yield were found to be $70 \%$ and $79 \%$, respectively.

\section{Particle size and morphology}

The particle size, size distribution, and zeta potential of gliadin nanoparticles were measured in a Zetasizer 3000 HS (Malvern Instrument Ltd., UK). Surface morphology and internal structure of gliadin nanoparticles were determined by scanning electron microscopy. A thin film of an aqueous dispersion of nanoparticles was applied on double stick tape over an aluminum stub and air-dried to get a uniform layer of particles. These particles were coated with gold to a thickness of about $450 \AA$ using sputter gold coater (Fig. 1).

\section{In vitro release studies}

The three different formulations were subjected to in vitro drug release studies in phosphate buffer saline of $\mathrm{pH} 7.4$ and phosphate buffer pH 6.8 medium using the biological membrane (135 HiMedia LA398). The observations are given in Table 1.

In vitro, drug release studies were conducted using Franz diffusion cell. Biological membrane was placed between the two chambers of cells. Phosphate buffer $\mathrm{pH} 7.4$ and 6.8 were used as a receptor diffusion media.

The dialysis system consisted of donor and receptor compartments separated by a biological membrane. The donor phase contained formulation equivalent to $10 \mathrm{mg}$ of drug-incorporated formulations, whereas the receptor phase contained phosphate buffer $\mathrm{pH} 7.4$ and $\mathrm{pH}$ 6.8. The medium volume $16 \mathrm{ml}$ was agitated at $100 \mathrm{rpm}$ with the help of magnetic stirrer at room temperature. Sink condition was maintained during drug release study. The samples were withdrawn at a specified time interval of $15 \mathrm{~min}$ and assayed spectrophotometrically at $\lambda_{\max } 227 \mathrm{~nm}$ (Shimadzu1700 UV/visible spectrophotometer) after making proper dilution. The drug release study was determined for $4 \mathrm{~h}$.

The \% drug release was calculated for all formulations, and the cumulative $\%$ drug release was plotted against time (Figs. 2 and 3 ).
In vivo studies

Animals; Balb/c is procured from NIN, Hyderabad, and inbred for experimental purposes in accordance with the CPCSEA guidelines, animals warehoused in individually ventilated cage system with $12 \mathrm{~h}$ light and dark cycle. The noise temperature and humidity were controlled, and as per the guidelines, animals were provided with sterile food water and bedding material.

Animals were administered $10 \mu \mathrm{l}$ of formulation (suspended in normal saline $1: 1$ ) in each nostril that is $20 \mu \mathrm{l}$ per animal. Animals were sacrificed at 15,30 , and $45 \mathrm{~min}$. Blood samples were collected by cardiac puncture, and brain tissue was collected and kept moist on ice. Brain tissue was homogenized as 1:1 ratio in normal saline solution. The homogenate was centrifuged at $1500 \mathrm{rpm}$ for $15 \mathrm{~min}$, and the supernatant $(1 \mathrm{ml})$ was precipitated and extracted with methanol. The plasma sample was also processed in a similar way except the homogenization step extract was centrifuged at $5000 \mathrm{rpm}$ step for 30 min and clear supernatant was analyzed on HPLC.

The ODS-3V C-18, $4.6 \times 250$ MM (GL SCIENCES Inc., Japan) chromatography column was used for analysis for sumatriptan. The mobile phase consisted of acetonitrile: water in a ratio of 80:20, respectively. The flow rate was $1 \mathrm{ml} / \mathrm{min}, \lambda_{\max } 227 \mathrm{~nm}, \mathrm{rt} 2.45 \mathrm{~min}$, and the results are shown in Table 2 and Fig. 4.

\section{DISCUSSION}

In the present work, sumatriptan-loaded gliadin nanoparticles were prepared and there selective targeting to the brain is done through nasal route. Sumatriptan given orally or deep rectally undergoes first-

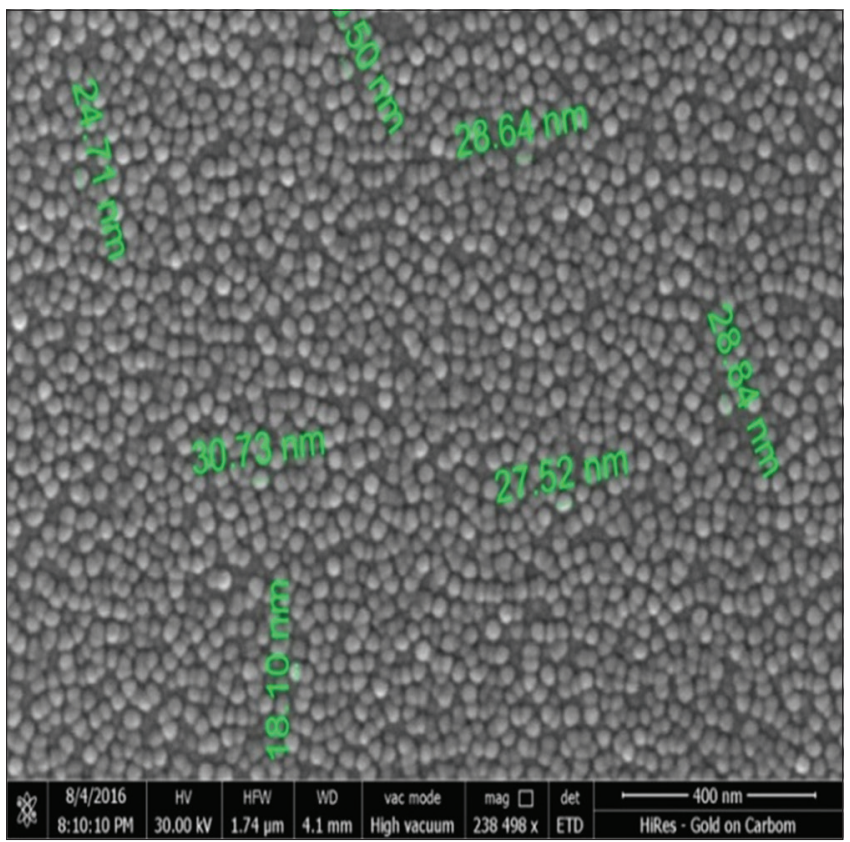

Fig. 1: Scanning electron microscopy photomicrograph of sumatriptan-loaded gliadin nanoparticles

Table 1: Specification of Franz diffusion cell

\begin{tabular}{lllll}
\hline S. No. & Specification & $\begin{array}{l}\text { Flat ground } \\
\text { joint }\end{array}$ & $\begin{array}{l}\text { Flat flange } \\
\text { joint }\end{array}$ & $\begin{array}{l}\text { “O” ring } \\
\text { joint }\end{array}$ \\
\hline 1. & Joint I.D & $15 \mathrm{~mm}$ & $15 \mathrm{~mm}$ & $15 \mathrm{~mm}$ \\
2. & Joint O.D & $30 \mathrm{~mm}$ & $30 \mathrm{~mm}$ & $35 \mathrm{~mm}$ \\
3. & Receptor & $15.5 \mathrm{ml}$ & $15.5 \mathrm{ml}$ & $15.5 \mathrm{ml}$ \\
& volume & & & \\
4. & Orifice area & $3.14 \mathrm{~cm}^{2}$ & $3.14 \mathrm{~cm}^{2}$ & $3.14 \mathrm{Cm}^{2}$ \\
5. & Jacket & $30 \mathrm{~mm}$ & $30 \mathrm{~mm}$ & $30 \mathrm{~mm}^{2}$ \\
& diameter & & & \\
\hline
\end{tabular}




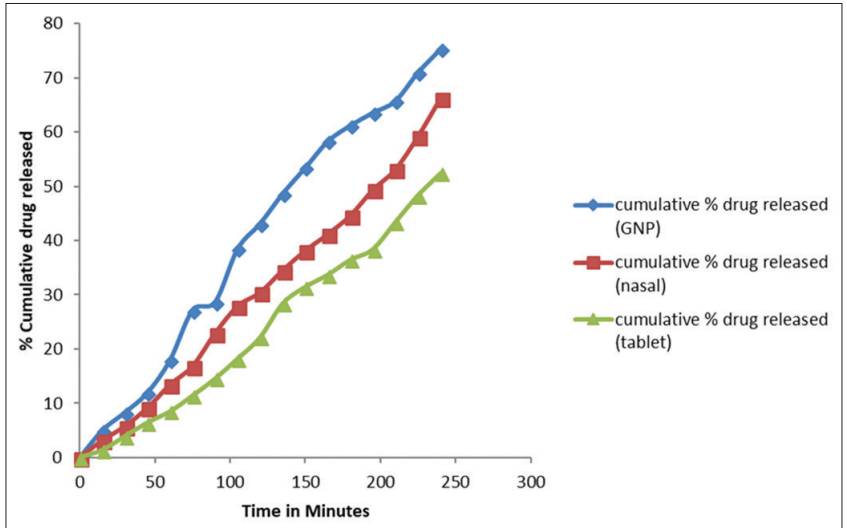

Fig. 2: Comparative percentage cumulative drug release profile of gliadin nanoparticles, marketed nasal spray, and tablet formulation at $6.8 \mathrm{pH}$

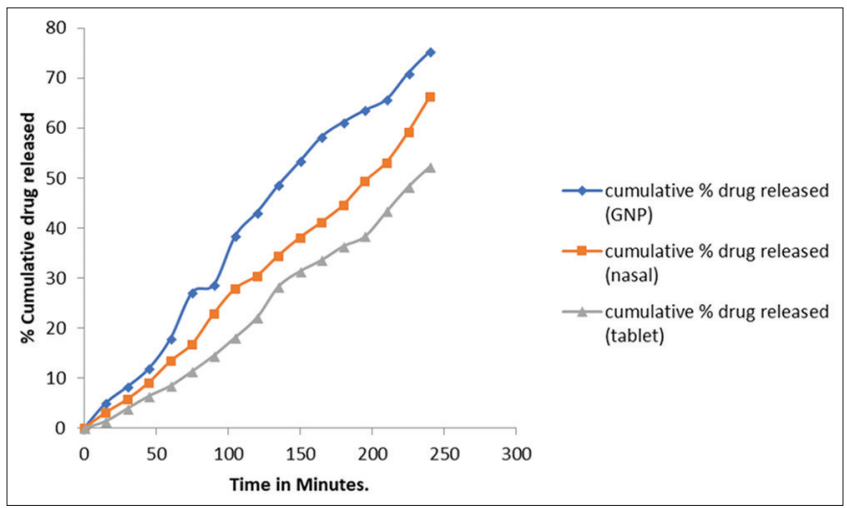

Fig. 3: Comparative percentage cumulative drug release profile of gliadin nanoparticles, marketed nasal spray, and tablet formulation at $7.4 \mathrm{pH}$

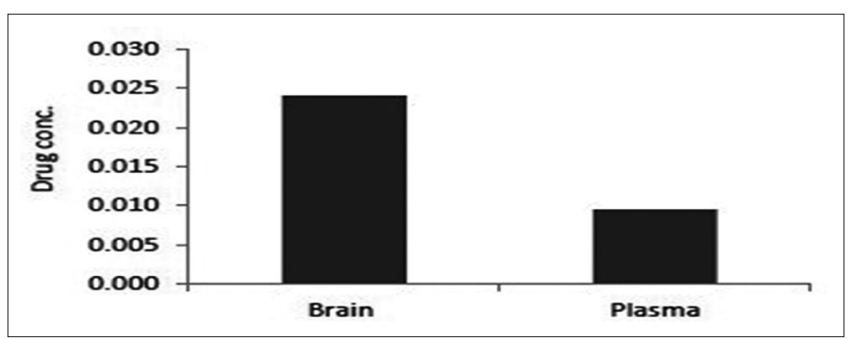

Fig. 4: Tissue drug concentration at 15 min post-administration (intranasal)

pass elimination that is the biotransformation of the drug within the gut lumen before absorption and within the intestinal epithelium and/ or liver after permeation of the intestinal mucosa is much higher as compared to nasal route. The optimized nanoparticles showed higher release rate than other marketed formulation like tablet, nasal spray, this may be due to nano size of the particles, glutaraldehyde was added to this system that increased hardness and F-68 increased the stability of nanoparticles, and gliadin showed good adhesive property due to their composition.

Optimized nanoparticles have showed fast action within 15 min in brain and plasma in vivo animal study of nasal mucosa, and blood samples were collected by cardiac puncture and brain tissue are analyzed during in vivo study.
Table 2: Tissue drug concentration at different time intervals after nasal administration

\begin{tabular}{llll}
\hline Time & Sample & AUC & Ug/ml \\
\hline 15 min & Brain & 232687 & 0.024 \\
& Plasma & 91874 & 0.010 \\
$30 \mathrm{~min}$ & Brain & Not detected & $\mathrm{Na}$ \\
& Plasma & Not detected & $\mathrm{Na}$ \\
$45 \mathrm{~min}$ & Brain & Not detected & $\mathrm{Na}$ \\
& Plasma & Not detected & $\mathrm{Na}$ \\
\hline
\end{tabular}

AUC: Area under curve

\section{CONCLUSION}

In this study, a gliadin-based nano drug delivery system for braintargeted delivery of sumatriptan through nasal route was prepared. First, the physiochemical properties of sumatriptan-loaded gliadin nanoparticles including the particles size and entrapment efficiency were carefully characterized. The results showed that sumatriptanloaded gliadin nanoparticles were effectively cross blood-brain barrier (BBB). The optimized nanoparticles showed higher release rate than other marketed formulation like a tablet, nasal spray, this may be due to nano size of the particles, glutaraldehyde was added to this system that increased hardness and F-68 increased the stability of nanoparticles, and gliadin showed good adhesive property due to their composition. Optimized nanoparticles have shown fast action within 15 min in brain and plasma. For in vivo animal study, the nasal mucosa and blood samples were collected by cardiac puncture and brain tissue were analyzed for the amount of drug. Gliadin nanoparticles are suitable for drug delivery of hydrophilic drugs, and they showed more than $80 \%$ bioavailability due to nanosizing of drug particles. Sumatriptan nanoparticles could be easy for intranasal administration. It overcame the problem of intranasal acceptability, physical instability, high manufacturing cost, and difficulties in scale up as a case of the intranasal formulation. The intranasal nasal route was selected due to the nearest targeted site to central nervous system, the absence of hepatic first pass, fast therapeutic effect, and the alternative to parentral route for brain drug delivery.

Due to higher hydrophilicity, sumatriptan is absorbed easily through olfactory mucosa and follow the transcellular pathway directly to the BBB, and this suitable action could be provided gliadin nanoparticles and sumatriptan in combination.

\section{CONFLICTS OF INTEREST}

The author(s) declare(s) that there is no conflict of interest.

\section{REFERENCES}

1. Goadsby PJ, Lipton RB, Ferrari MD. Migraine-current understanding and treatment. N Engl J Med 2002;346:257-70.

2. Farshid A, Rama B, Gopal Muralidharan NL. Formulation and evaluation of orodispersible tablets of zolmitriptan. Asian J Pharm Clin Res 2014;7:127-34.

3. Ferrari MD, Roon KI, Lipton RB, Goadsby PJ. Oral triptans (serotonin 5-HT1B/1D agonists) in acute migraine treatment: A meta-analysis of 53 trials. Lancet 2001;358:1668-75.

4. Lipton RB, Diamond S, Reed M, Diamond ML, Stewart WF. Migraine diagnosis and treatment: Results from the American Migraine Study II. Headache J Head Face Pain 2001;41:638-45.

5. Humphrey PP, Feniuk W. Mode of action of the anti-migraine drug sumatriptan. Trends Pharmacol Sci 1991;12:444-6.

6. Chavan VR, Mudium RA, Sayana SB. Abolition of seizure provoking effect of sumatriptan by fluoxetine in pentylenetetrazol induced seizures in rats. Asian J Pharm Clin Res 2014;7:107-9.

7. Ramteke S, Maheshwari RU, Jain NK. Clarithromycin based oral sustained release nanoparticulate drug delivery system. Indian J Pharm Sci 2006;68:474-84. 